\title{
Regional Difference in the Association between the Trajectory of Selenium Intake and Hypertension: A 20-Year Cohort Study
}

\author{
Changxiao Xie ${ }^{1,2,3} \mathbb{D}$, Jinli Xian ${ }^{1,2,3}$, Mao Zeng ${ }^{1,2,3}$, Zhengjie Cai ${ }^{1,2,3}$, Shengping Li ${ }^{1,2,3}$, Yong Zhao ${ }^{1,2,3,4,5, * \mathbb{D}}$ \\ and Zumin Shi ${ }^{6}$ (D)
}

1 Department of Nutrition and Food Hygiene, School of Public Health and Management, Chongqing Medical University, Chongqing 400016, China; 2019110926@stu.cqmu.edu.cn (C.X.); 2018111037@stu.cqmu.edu.cn (J.X.); zengmao@stu.cqmu.edu.cn (M.Z.); 2018111015@stu.cqmu.edu.cn (Z.C.); 2019110981@stu.cqmu.edu.cn (S.L.)

2 Research Center for Medicine and Social Development, Chongqing Medical University, Chongqing 400016, China

3 The Innovation Center for Social Risk Governance in Health, Chongqing Medical University, Chongqing 400016, China

4 Chongqing Key Laboratory of Child Nutrition and Health, Children's Hospital of Chongqing Medical University, Chongqing 400014, China

5 School of Public Health and Management, Chongqing Medical University, Yixueyuan Road, Yuzhong District, Chongqing 400016, China

6 Human Nutrition Department, College of Health Sciences, QU Health, Qatar University, Doha 2713, Qatar; zumin@qu.edu.qa

* Correspondence: zhaoyong@cqmu.edu.cn; Tel.: +86-138-8346-0842; Fax: +86-23-68485031

Citation: Xie, C.; Xian, J.; Zeng, M.; Cai, Z.; Li, S.; Zhao, Y.; Shi, Z. Regional Difference in the Association between the Trajectory of Selenium Intake and Hypertension: A 20-Year Cohort Study. Nutrients 2021, 13, 1501. https://doi.org/10.3390/nu13051501

Academic Editors: Lutz Schomburg and Maria Luz Fernandez

Received: 24 March 2021

Accepted: 27 April 2021

Published: 29 April 2021

Publisher's Note: MDPI stays neutral with regard to jurisdictional claims in published maps and institutional affiliations.

Copyright: (c) 2021 by the authors. Licensee MDPI, Basel, Switzerland. This article is an open access article distributed under the terms and conditions of the Creative Commons Attribution (CC BY) license (https:// creativecommons.org/licenses/by/ $4.0 /)$.
Abstract: The effect of selenium on hypertension is inconclusive. We aimed to study the relationship between selenium intake and incident hypertension. Adults (age $\geq 20$ years) in the China Health and Nutrition Survey were followed up from 1991 to $2011(N=13,668)$. The latent class modeling method was used to identify trajectory groups of selenium intake. A total of 4039 respondents developed hypertension. The incidence of hypertension was 30.1, 30.5, 30.6, and 31.2 per 1000 person-years among participants with cumulative average selenium intake of $21.0 \pm 5.1,33.2 \pm 2.8,43.8 \pm 3.6$, and $68.3 \pm 25.2 \mu \mathrm{g} /$ day, respectively. Region and selenium intake interaction in relation to hypertension was significant. In the multivariable model, cumulative intake of selenium was only inversely associated with the incident hypertension in northern participants (low selenium zone), and not in southern participants. Compared to selenium intake trajectory Group 1 (stable low intake), all three trajectory groups had a low hazard ratio for hypertension among the northern participants. However, Group 4 (high intake and decreased) showed an increasing trend of hypertension risk in the south. In conclusion, the association between selenium intake and the incidence of hypertension varied according to regions in China. In the low soil selenium zone, high selenium intake might be beneficial for hypertension prevention.

Keywords: selenium intake; hypertension; cohort study; china health; nutrition survey

\section{Introduction}

Hypertension is one of the most critical risk factors for cardiovascular disease and even mortality. In 2014 , the prevalence of hypertension in adults (aged $\geq 18$ years) was approximately $22 \%$ worldwide [1]. According to the China Hypertension Survey results in 2012-2015, 245 million adults (23.2\% of the population) suffered from hypertension [2]. Dietary factors contribute significantly to blood pressure [3].

Selenium (Se) is an essential trace element for human health. In selenoprotein, it plays a variety of physiological functions on the body, such as antioxidant activity, prevention of viral mutations, protection of intestinal mucosa, anti-inflammatory activity, glucose metabolism effects, and insulin sensitivity effects [4]. The organic forms of selenium found 
in human body are selenocystein and selenoproteins. Seleproteins are known as antioxidants, which protect cells from the damage caused by free radicals. Glutathione peroxidase (GPX) is the major selenoprotein in the human body [5]. Selenium deficiency leads primarily to degeneration of many organs and tissues, and has been linked to the risk of Keshan disease, Kashin-Beck disease, cancer, cardiovascular disease and even AIDS [5,6]. Worldwide, $1 / 7$ people are on a low-selenium diet [7]. China is among the countries heavily affected by selenium deficiency [8]. A study of residents in 10 provinces in China showed that their selenium levels decreased by $24-46 \%$ over the past two decades due to decreased grain consumption and low selenium content in rice [8].

Selenium affects the prevention and treatment of hypertension because of its antioxidant capacity $[9,10]$. Many studies showed a correlation between selenium and blood pressure. One review published in 2014 included 25 studies with more than 26,000 participants from 19 countries. Half of the 25 studies did not show any relationship between selenium and hypertension, whereas the other half found either a positive or a negative association [11]. Another systematic review published in 2019 identified six studies on the association between selenium intake and metabolic syndrome, and the association was found to be inconclusive [12]. These conflicting findings may be because selenium status is positively related to the different study populations' soil selenium levels. Selenium has a safe narrow window. Inorganic sources of selenium exhibit higher toxicity, as compared to the organic forms [5]. A massive overdose of selenium intake may have toxic effects and cause various adverse effects, including diarrhea, hair loss, nausea, headache, nervous system disorders, and death [13].

Most present studies on the relationship between selenium status and hypertension used serum selenium concentration as the indicator of selenium status [11]. Few population studies have explored the relationship between selenium intake and hypertension. No longitudinal study has investigated the association between selenium intake and hypertension.

Using data from the China Health and Nutrition Survey (CHNS), we wanted to evaluate the association between selenium intake and hypertension. Furthermore, we aimed to assess the selenium intake trajectory in the general population and its association with hypertension.

\section{Methods}

\subsection{Study Sample}

The CHNS is a prospective cohort study reviewed by the University of North Carolina (USA) and the National Institute of Nutrition and Food Safety (China). This study was designed to monitor the Chinese population's health and nutrition status using a multistage random cluster sampling method to survey nine provinces of China. Geographically, we defined Heilongjiang, Liaoning, Shandong, and Henan as the northern regions and Jiangsu, Hubei, Hunan, Guizhou, and Guangxi as the southern regions. The respondents in the cohort included all the family members of the selected family. By 2011, the CHNS had conducted nine surveys (1989, 1991, 1993, 1997, 2000, 2004, 2006, 2009, and 2011). The 1989 survey did not collect data for each age group. Thus, we only analyzed the data collected from 1991 to 2011. Due to the high loss to follow-up caused by the accelerated urbanization process, residents of new communities were invited to participate in the survey as supplementary samples since 1997. The response rate for respondents with informed consent from 1989 to 2006 was more than 60 percent. In eight surveys from 1991 to $2011,29,220$ participants aged $\geq 20$ years old were included in the study. We excluded subjects who had no dietary intake information $(n=4982)$, those who had incredible estimates of their daily energy intake (males: $>6000 \mathrm{kcal}$ or $<800 \mathrm{kcal}$; females: $>4000$ or $<600 \mathrm{kcal})(n=113)$, pregnant or breastfeeding females $(n=169)$, individuals with implausible BMI (Body Mass Index, $<14$ or $>45 \mathrm{~kg} / \mathrm{m}^{2}, n=7$ ), and those who participated in only one wave $(n=8470)$. A total of 15,479 samples were used as a baseline, and their selenium intake was estimated. Focusing on newly discovered hypertension at follow-up, we excluded patients who had hypertension at baseline $(n=1811) .13,668$ samples were 
finally analyzed. Those excluded in the analysis were older (44.5 vs. 39.2 years), had high BMI (23.3 vs. 22.0$)$ and were more likely to have high income ( $44.8 \%$ vs. $37.5 \%$ ) and live in the urban area ( $54.0 \%$ vs. $34.4 \%)$.

In the selenium intake trajectory analyses, we included participants who completed at least three waves of dietary intake $(N=11,194)$, including those with hypertension at baseline. After excluding those with hypertension at baseline $(n=1169)$, the remaining 10,025 participants were included in the analysis of the association between the trajectory of selenium intake and incident hypertension (Figure 1).

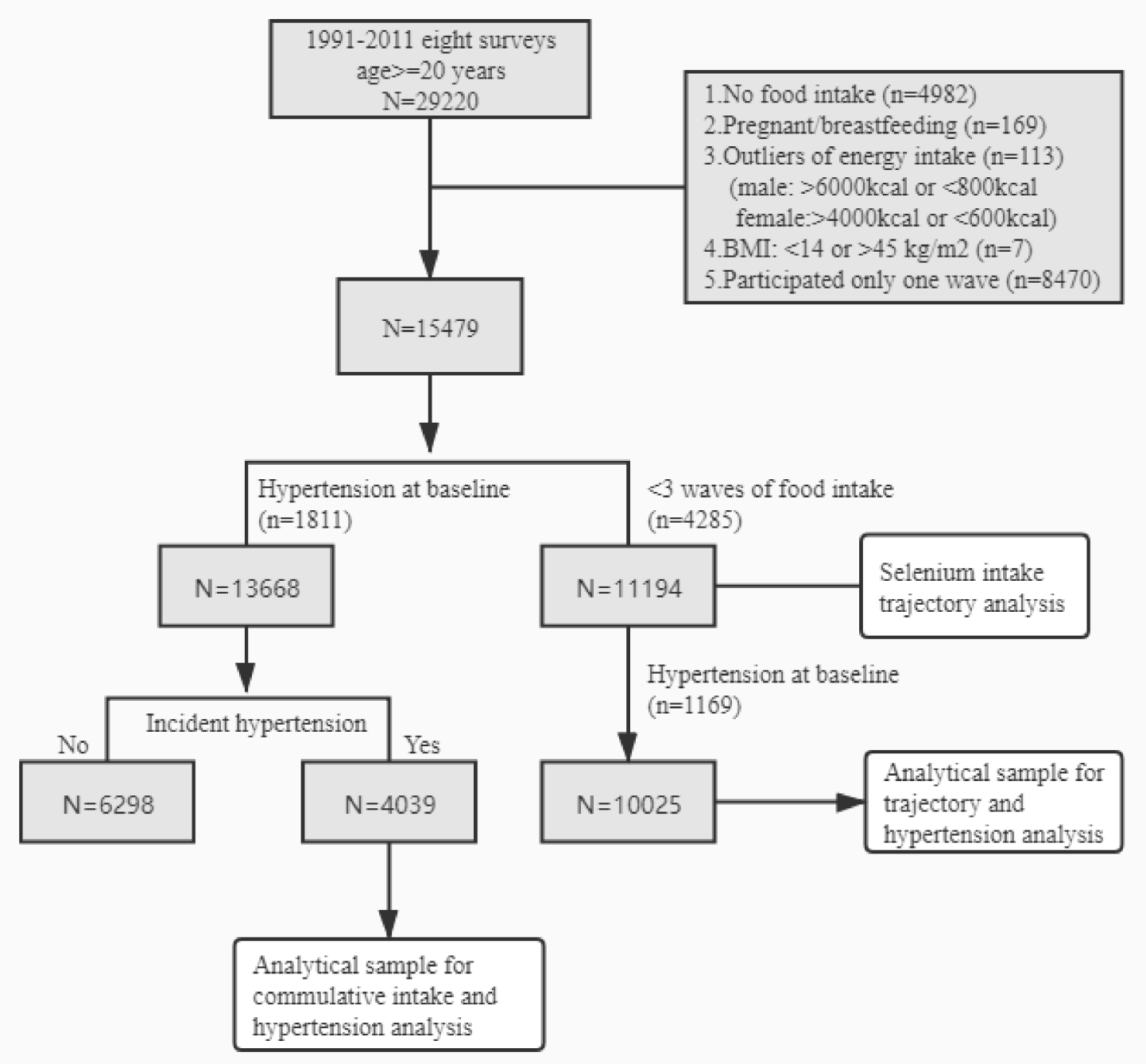

Figure 1. Participant flow chart.

\subsection{Outcome Variable: Hypertension}

This part was performed by doctors or well-trained medical workers in a community service center. Participants were told to rest for $10 \mathrm{~min}$ and then have their blood pressure measured while sitting. Trained staff used a mercury sphygmomanometer (wellcalibrated) to measure blood pressure in participants' right arms. Three measurements were made for each person, and the average value was considered to be the final value. For patients with diagnosed hypertension, we measured their blood pressure before taking medication. Participants were labeled as having hypertension when their average systolic blood pressure $>140 \mathrm{mmHg}$ and diastolic blood pressure $>90 \mathrm{mmHg}$ or being diagnosed with hypertension. 


\subsection{Exposure Variables: Selenium Intake}

During the three-day diet survey, trained investigators weighed and recorded food and condiments from household stock, market, garden, and leftover food waste. According to China Food Composition Tables, the intake of nutrients, including selenium and protein, was collected based on the average of three-day food consumption information. Regional differences of nutrient content in food (e.g., rice) grown in different regions were reflected in the China Food Composition Table by assigning different food codes [14]. We calculated the cumulative selenium intake and used it as an exposure variable. This treatment effectively contributed to the reduced variation among individuals and represented long-term eating habits [15]. For example, if a person's intake was $x$ in 1991, y in 1993 and $z$ in 1997, $(x+y) / 2$ was the cumulative mean in 1993 and $(x+y+z) / 3$ was the cumulative mean in 1997. The baseline intake was $x$ and the most recent intake for 1991, 1993 and 1997 was $x, y$ and $z$, respectively. Furthermore, we assigned quartiles based on all the observations.

\subsection{Covariates}

In each survey, sociodemographic characteristics and health-related lifestyle information must be obtained through questionnaires. The socioeconomic variables were as follows: education (low, illiterate/primary school; medium, junior middle school; and high, high middle school and higher); per capita annual family income (low, medium, and high); and urbanization levels (low, medium, and high) [16]. The metabolic equivalent was estimated based on self-reported activities (including occupation, family, transportation, and recreation) and time spent and was used to reflect physical activity levels. Alcohol intake options were set as none, 1-2 times/week, 3-4 times/week, and daily. Classification variables were used to describe smoking status: non-smokers, ex-smokers, and current smokers. Overweight/obesity was defined as BMI $>24 \mathrm{~kg} / \mathrm{m}^{2}$ (Chinese standards) $[17,18]$. Meanwhile, we calculated the prevalence of diabetes in each group.

\subsection{Statistical Analysis}

Selenium intake was categorized into quartiles. The Chi-square test was adopted to analyze the differences of classification variables. The one-way analysis of variance was used to compare the differences of continuous variables. Cox proportional hazard model with time-varying cumulative selenium intake and covariates was used to calculate the hazard ratio (HR) for incident hypertension. Three models were established, as follows. Model 1 was adjusted for age, sex, and energy intake. Model 2 was further adjusted for fat and sodium intake, smoking, alcohol consumption, income, urbanization, education, physical activity, and BMI. Model 3 was further adjusted for two dietary patterns: the traditional southern and the modern patterns, based on a previously published factor analysis [19]. The traditional southern eating pattern is high in rice, pork, and vegetables and low in wheat. The modern diet is heavy on fruit, soy milk, eggs, milk, and fried foods. Cox proportional hazards model assumptions were examined by visual inspection of log$\log$ plots. Multiplicity interaction between selenium intake and demographic characteristics (sex, income, education, and residence) was analyzed by adding the product of variables in the regression model.

The latent class modeling method was applied to trajectory analysis. We constructed a group-based trajectory modeling to identify distinctive subgroups of individual trajectories of selenium intake within a population and profiling characteristics of individuals within the subgroups using the Stata command, traj. In the analysis, we included all participants who had data on their dietary intake collected at least three waves during 1991 and 2011. For each trajectory, we estimated several possible combinations of trajectory shapes (intercept only, linear, quadratic or cubic) to identify the model. The number of trajectory groups was finally determined based on two criteria: (1) the smallest value of Bayesian information criterion and (2) each group should have at least $3 \%$ of participants. Finally, the Cox proportional hazards model was adopted to analyze the relationship between selenium intake and incident hypertension risk. Model was adjusted for age, sex, intake 
of energy, fat and sodium, smoking, alcohol drinking, income, urban, education, physical activity, and BMI. The results were visually presented.

STATA 16.1 was used. Statistical significance would be identified when $p<0.05$ (two-sided).

\section{Results}

At baseline, participants in the highest quartile of cumulative selenium intake had higher income and BMI than those in the lowest quartile (Table 1). Energy and sodium intake were positively associated with selenium intake. In addition, smokers were more likely to have higher selenium intake than the others. Most of the participants had baseline selenium intake below $60 \mu \mathrm{g} /$ day (Supplement Figure S1). The participants from the north had higher selenium intake than those from the south. At baseline before excluding those with hypertension, across quartiles of Se intake, the prevalence of hypertension was $13.2 \%$, $12.0 \%, 12.3 \%$ and $13.7 \%$ in the whole sample; $11.3 \%, 10.4 \%, 11.7 \%$ and $11.5 \%$ in the south; and $18.0 \%, 14.4 \%, 13.7 \%$ and $15.6 \%$ in the north.

Table 1. Baseline sample characteristics by quartiles of Se intake: CHNS $(N=13,668)$.

\begin{tabular}{|c|c|c|c|c|c|}
\hline Factor & Q1 & Q2 & Q3 & Q4 & $p$-Value \\
\hline$N$ & 3453 & 3385 & 3423 & 3407 & \\
\hline Se intake ( $\mu \mathrm{g} /$ day), mean (SD) & $21.0(5.1)$ & $33.2(2.8)$ & $43.8(3.6)$ & $68.3(25.2)$ & $<0.001$ \\
\hline Energy intake (kcal/day), mean (SD) & $2054.1(588.7)$ & $2329.0(557.9)$ & $2572.3(611.3)$ & $2902.0(759.2)$ & $<0.001$ \\
\hline Fat intake (g/day), mean (SD) & $49.7(29.2)$ & $60.8(32.8)$ & $68.7(35.4)$ & $85.2(42.5)$ & $<0.001$ \\
\hline Protein intake (g/day), mean (SD) & $54.6(15.5)$ & $66.9(15.0)$ & $77.8(17.4)$ & $94.8(24.9)$ & $<0.001$ \\
\hline Carbohydrate intake (g/day), mean (SD) & $344.3(120.2)$ & $375.1(118.6)$ & $405.4(137.1)$ & $431.3(164.2)$ & $<0.001$ \\
\hline Sodium intake (g/day), mean (SD) & $5.7(6.5)$ & $5.9(4.9)$ & $6.4(4.7)$ & $7.3(5.6)$ & $<0.001$ \\
\hline Traditional dietary pattern, mean (SD) & $0.0(0.8)$ & $-0.1(1.0)$ & $-0.1(1.1)$ & $0.0(1.4)$ & $<0.001$ \\
\hline Modern dietary pattern, mean (SD) & $-0.6(0.5)$ & $-0.4(0.7)$ & $-0.2(0.8)$ & $0.3(1.1)$ & $<0.001$ \\
\hline Age (years), mean (SD) & $40.7(15.6)$ & $39.7(14.6)$ & $38.5(13.5)$ & $37.8(13.1)$ & $<0.001$ \\
\hline BMI $\left(\mathrm{kg} / \mathrm{m}^{2}\right)$, mean $(\mathrm{SD})$ & $21.5(2.8)$ & $21.8(2.8)$ & $22.2(2.9)$ & $22.4(3.0)$ & $<0.001$ \\
\hline $\mathrm{BMI} \geq 24\left(\mathrm{~kg} / \mathrm{m}^{2}\right)$ & $17.6 \%$ & $19.4 \%$ & $24.2 \%$ & $26.0 \%$ & $<0.001$ \\
\hline Sex & & & & & $<0.001$ \\
\hline Males & $38.1 \%$ & $44.3 \%$ & $51.3 \%$ & $61.5 \%$ & \\
\hline Females & $61.9 \%$ & $55.7 \%$ & $48.7 \%$ & $38.5 \%$ & \\
\hline Income & & & & & $<0.001$ \\
\hline Low & $39.5 \%$ & $32.0 \%$ & $26.0 \%$ & $21.7 \%$ & \\
\hline Medium & $33.3 \%$ & $33.9 \%$ & $33.4 \%$ & $30.1 \%$ & \\
\hline High & $27.1 \%$ & $34.1 \%$ & $40.6 \%$ & $48.1 \%$ & \\
\hline Education & & & & & $<0.001$ \\
\hline Low & $55.0 \%$ & $47.7 \%$ & $41.1 \%$ & $35.0 \%$ & \\
\hline Medium & $30.5 \%$ & $32.4 \%$ & $33.5 \%$ & $36.3 \%$ & \\
\hline High & $14.5 \%$ & $19.9 \%$ & $25.3 \%$ & $28.7 \%$ & \\
\hline Diabetes & $4.7 \%$ & $6.4 \%$ & $7.2 \%$ & $8.6 \%$ & 0.55 \\
\hline Urbanization & & & & & $<0.001$ \\
\hline Low & $49.4 \%$ & $42.3 \%$ & $38.7 \%$ & $32.4 \%$ & \\
\hline Medium & $29.5 \%$ & $29.8 \%$ & $29.1 \%$ & $30.3 \%$ & \\
\hline High & $21.1 \%$ & $27.9 \%$ & $32.2 \%$ & $37.3 \%$ & \\
\hline Smoking & & & & & $<0.001$ \\
\hline Non smoker & $72.0 \%$ & $68.8 \%$ & $65.2 \%$ & $59.0 \%$ & \\
\hline Ex-smokers & $1.8 \%$ & $1.5 \%$ & $1.6 \%$ & $1.8 \%$ & \\
\hline Current smokers & $26.2 \%$ & $29.7 \%$ & $33.2 \%$ & $39.2 \%$ & \\
\hline Physical activity (MET-hrs/week), mean (SD) & $208.8(175.2)$ & $211.0(182.9)$ & $206.4(180.3)$ & $191.7(156.0)$ & $<0.001$ \\
\hline
\end{tabular}


During a median follow-up of nine years, 4039 of 13,688 participants (for a total of 132,087 person-years) developed hypertension. Across the cumulative selenium intake quartiles, hypertension incidence was 30.1, 30.5, 30.6, and 31.2 per 1000 person-years (Table 2). In the fully adjusted model, across the quartiles of cumulative selenium intake, the HRs for incident hypertension were: 1.00, 0.91 (95\% CI 0.82-1.01), 0.90 (95\% CI 0.80$1.00)$, and 0.96 (95\% CI 0.85-1.08). There was a significant positive association between the most recent selenium intake and hypertension. However, the association was attenuated and deemed statistically significant after further adjusting for dietary patterns. Baseline selenium intake was not associated with hypertension.

Table 2. Hazard ratio (95\% CI) for incident hypertension by quartiles of Se intake: CHNS $(N=4039)$.

\begin{tabular}{|c|c|c|c|c|c|}
\hline & \multicolumn{5}{|c|}{ Se Intake (g/Day) } \\
\hline & Q1 & Q2 & Q3 & Q4 & $p$ for Trend \\
\hline \multicolumn{6}{|c|}{ A. Cumulative average Se intake } \\
\hline Cases & 1028 & 1002 & 1005 & 1004 & \\
\hline Person-years & 34,193 & 32,834 & 32,891 & 32,169 & \\
\hline Incident rate (per 1000) & 30.1 & 30.5 & 30.6 & 31.2 & \\
\hline Model 1 & 1.00 & $0.98(0.90-1.07)$ & $1.03(0.94-1.12)$ & $1.19(1.09-1.31)$ & $<0.001$ \\
\hline Model 2 & 1.00 & $0.92(0.83-1.02)$ & $0.93(0.83-1.03)$ & $1.01(0.90-1.13)$ & 0.861 \\
\hline Model 3 & 1.00 & $0.91(0.82-1.01)$ & $0.90(0.80-1.00)$ & $0.96(0.85-1.08)$ & 0.445 \\
\hline \multicolumn{6}{|l|}{ B. Baseline Se intake } \\
\hline Cases & 1056 & 962 & 1039 & 982 & \\
\hline Person-years & 34,317 & 33,326 & 33,265 & 31,179 & \\
\hline Incident rate (per 1000) & 30.8 & 28.9 & 31.2 & 31.5 & \\
\hline Model 1 & 1.00 & $0.97(0.89-1.05)$ & $1.10(1.01-1.20)$ & $1.15(1.05-1.25)$ & $<0.001$ \\
\hline Model 2 & 1.00 & $0.93(0.84-1.03)$ & $1.00(0.90-1.10)$ & $1.00(0.90-1.11)$ & 0.672 \\
\hline Model 3 & 1.00 & $0.92(0.83-1.01)$ & $0.97(0.88-1.07)$ & $0.97(0.87-1.07)$ & 0.762 \\
\hline \multicolumn{6}{|l|}{ C. Most recent Se intake } \\
\hline Cases & 1050 & 942 & 1023 & 1024 & \\
\hline Person-years & 33,568 & 33,011 & 33,348 & 32,160 & \\
\hline Incident rate (per 1000) & 31.3 & 28.5 & 30.7 & 31.8 & \\
\hline Model 1 & 1.00 & $1.02(0.93-1.12)$ & 1.17 (1.07-1.28) & $1.27(1.15-1.40)$ & $<0.001$ \\
\hline Model 2 & 1.00 & $0.93(0.84-1.03)$ & $1.03(0.93-1.15)$ & $1.11(0.99-1.24)$ & 0.030 \\
\hline Model 3 & 1.00 & $0.92(0.83-1.02)$ & $1.01(0.91-1.13)$ & $1.08(0.95-1.22)$ & 0.121 \\
\hline
\end{tabular}

Model 1 adjusted for age, sex and energy intake, Model 2 further adjusted for intake of fat and sodium, smoking, alcohol drinking, income, urban, education, physical activity and BMI. Model 3 further adjusted for dietary patterns. All the adjusted variables are treated as time-varying covariates. Bold: statistically significant.

No interactions were observed between selenium intake and sex, income, and urbanization level to hypertension risk (Table 3). A significant selenium and region interaction was observed. In northern participants, cumulative selenium intake was inversely associated with hypertension risk. However, no such association was found in participants from the south. In contrast, high selenium intake tends to be positively associated with hypertension in participants from the south.

In group-based trajectory analysis of selenium intake between 1991 and 2011, four groups were identified (Figure 2) as follows: Group 1 (stable and low, $n=7149,67.0 \%$ ) had lower-than-average selenium intake profiles during all the waves; Group 2 (stable and medium, $n=2364,26.3 \%$ ) had a stable medium intake of Selenium; Group 3 (increasing and medium, $n=299,3.7 \%$ ) had a medium and increasing selenium intake; and Group 4 (descending and high, $n=213,3.0 \%$ ) had a high intake that decreased over time. The mean baseline selenium intakes in Groups 1, 2, 3, and 4 were 35.7, 51.2, 51.2, and $87.6 \mu \mathrm{g} /$ day, respectively (Supplement Table S1). There were significant differences in all sociodemographic characteristics and health-related lifestyles among the four groups. Group 4 participants were more likely to be males with high income and education than those in other groups. 
Table 3. Subgroup analyses of the association between cumulative intake and incident hypertension: CHNS (1991-2011).

\begin{tabular}{|c|c|c|c|c|c|}
\hline & Q1 & Q2 & Q3 & Q4 & $p$ for Interaction \\
\hline \multicolumn{6}{|l|}{ BMI status } \\
\hline Normal & 1.00 & $0.92(0.81-1.04)$ & $0.88(0.77-1.02)$ & $1.03(0.88-1.21)$ & \multirow[t]{2}{*}{0.496} \\
\hline Overweight/obese & 1.00 & $0.88(0.74-1.05)$ & $0.92(0.78-1.10)$ & $0.89(0.74-1.07)$ & \\
\hline \multicolumn{6}{|l|}{ Region } \\
\hline North & 1.00 & $0.94(0.80-1.11)$ & $0.82(0.69-0.97)$ & $0.84(0.70-1.00)$ & \multirow[t]{2}{*}{0.001} \\
\hline South & 1.00 & $0.89(0.77-1.03)$ & $0.96(0.82-1.13)$ & $1.11(0.93-1.33)$ & \\
\hline \multicolumn{6}{|l|}{ Income } \\
\hline Low & 1.00 & $0.84(0.71-1.00)$ & $0.91(0.75-1.10)$ & 0.87 (0.69-1.09) & \multirow[t]{3}{*}{0.541} \\
\hline Medium & 1.00 & $0.97(0.81-1.15)$ & 0.89 (0.73-1.07) & $0.96(0.78-1.18)$ & \\
\hline High & 1.00 & $0.94(0.78-1.13)$ & $0.94(0.78-1.13)$ & $1.03(0.85-1.26)$ & \\
\hline \multicolumn{6}{|l|}{ Sex } \\
\hline Males & 1.00 & $0.84(0.71-0.99)$ & $0.85(0.72-1.00)$ & $0.91(0.77-1.08)$ & \multirow[t]{2}{*}{0.677} \\
\hline Females & 1.00 & $0.97(0.85-1.11)$ & $0.94(0.81-1.09)$ & $0.99(0.84-1.18)$ & \\
\hline \multicolumn{6}{|l|}{ Urbanization level } \\
\hline Low & 1.00 & $0.83(0.70-0.99)$ & $0.95(0.80-1.14)$ & $0.94(0.76-1.16)$ & \multirow[t]{3}{*}{0.241} \\
\hline Medium & 1.00 & $0.88(0.74-1.04)$ & $0.81(0.68-0.98)$ & $0.98(0.80-1.19)$ & \\
\hline High & 1.00 & $1.08(0.88-1.32)$ & $0.98(0.80-1.21)$ & $1.02(0.82-1.27)$ & \\
\hline
\end{tabular}

Model adjusted for age, sex, intake of energy, fat and sodium, smoking, alcohol drinking, income, urban, education, physical activity, and BMI, and dietary patterns. Stratification variables were not adjusted in the corresponding models. Bold: statistically significant.

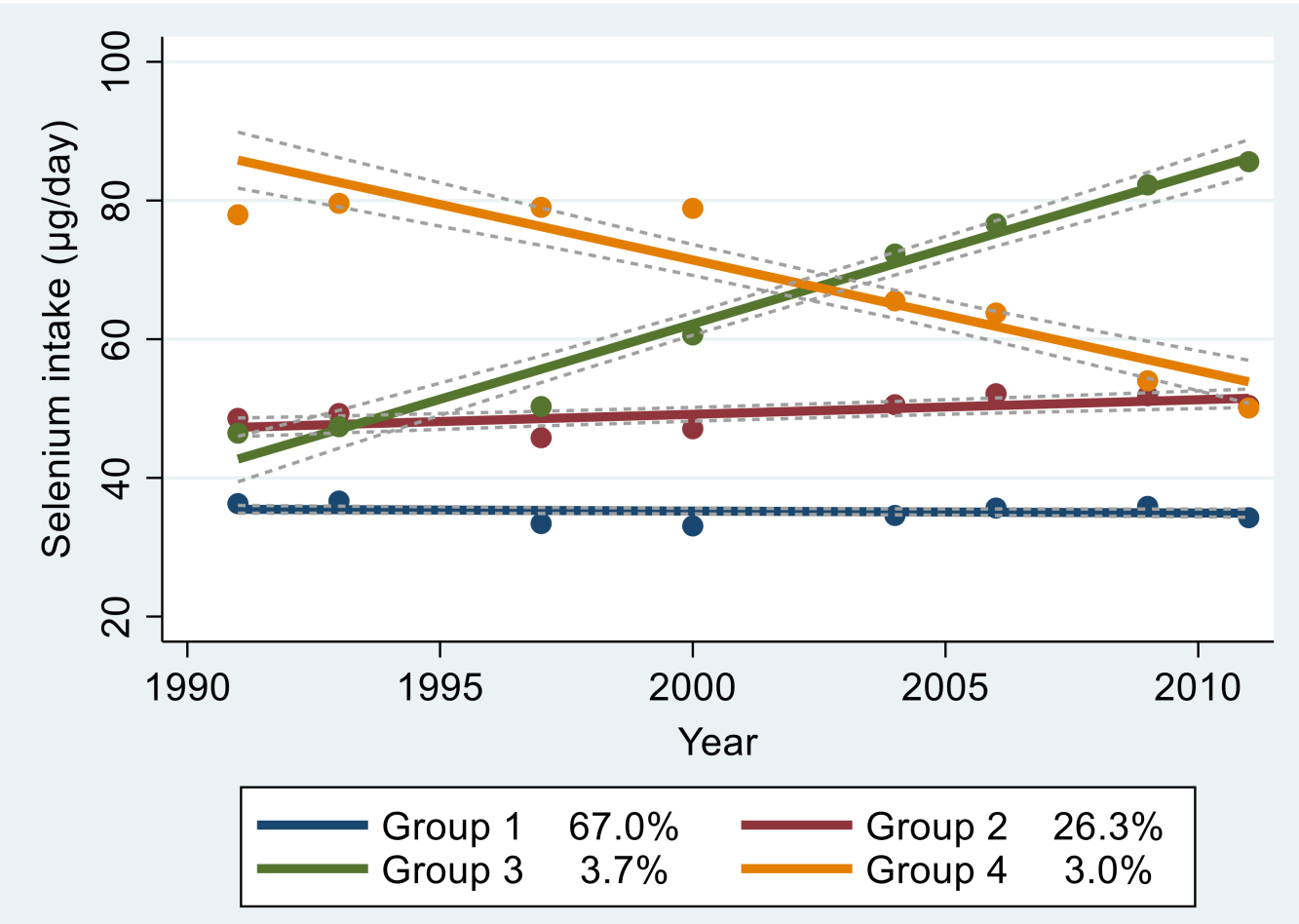

Figure 2. Group based trajectory of Se intake among adults attending the China Health and Nutrition Survey (CHNS), 1991-2011. ( $=11,194)$.

Group 1 had stable low intake of Se. Group 2 had stable medium intake of Se. Group 3 had me dium Se intake and increased. Group 4 had a high intake and decreased. Dash lines represent 95\% CI. Figure 3 shows the opposite associations between selenium intake trajectory groups in participants from the south and north (Group 1 was used as 
reference). In participants from the north, Groups 2, 3, and 4 were positively associated with incident hypertension with HRs of 0.84 (95\% CI 0.74-0.95), 0.72 (95\% CI 0.53-0.99), and 0.63 (95\% CI 0.44-0.89), respectively. However, in participants from the south, the corresponding figures were 1.01 (95\% CI 0.87-1.17), 1.34 (95\% CI 0.95-1.88), and 2.09 (95\% CI 1.37-3.18), respectively.

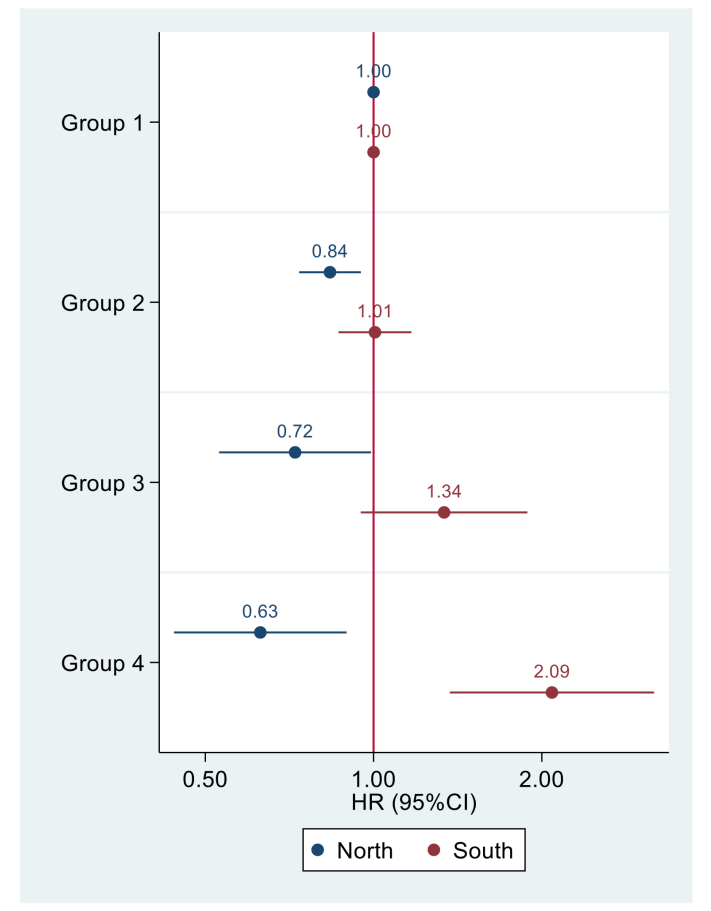

Figure 3. Association between trajectory of Se intake and incident hypertension among adults attending in CHNS.

Model adjusted for age, sex, intake of energy, fat and sodium, smoking, alcohol drinking, income, urban, education, physical activity, and BMI.

Trajectory pattern was modeled using traj command in Stata. Participants had at least three waves of selenium intake. Group 1 (stable low intake) was used as the reference in multivariable Cox regression.

\section{Discussion}

In this large prospective cohort study, the selenium intake of most of the participants was below the recommended nutrient intake $(60 \mu \mathrm{g} /$ day) set by the Chinese Nutrition Society [20], and none of the participants had a selenium intake that is above the tolerable upper intake level (UL) of $400 \mu \mathrm{g} /$ day. The cumulative average selenium intake was inversely associated with the incidence of hypertension in participants from the north but not those from the south; it was associated with overall dietary patterns. The selenium intake trajectory analysis suggested that the intake of selenium was relatively stable in the majority of the samples; only a small proportion had a slight increase in intake (3.7\%) or decrease in intake (3.0\%) over 20 years. This was different from the results obtained by Li et al. [8] that the overall hair selenium content of inhabitants lower $24-46 \%$ than they were 20 years ago. It was due to different research methods. We divided the selenium intake into four categories according to different selenium intake trajectories without investigating overall selenium intake.

To the best of our knowledge, this is the first longitudinal study that examines the association between selenium intake and hypertension. A cohort study conducted in Western Europe showed that low blood selenium was a risk factor for hypertension in males [21]. Western Europe is generally low in selenium, similar to the northern regions in China mentioned in this study. Two longitudinal studies in China examined the association 
between selenium status and incident hypertension. In a study conducted in older Chinese adults in Sichuan and Shandong Provinces, nail selenium was positively associated with incident hypertension risk [22]. However, the study excluded participants from regions with endemic diseases, including Keshan disease.

In contrast, using a sub-cohort of 2530 adults who attended the CHNS between 2009 and 2015, low serum selenium level was related to the increased risk of developing hypertension [23]. Comparing the extreme quartiles of serum selenium, the relative risk for hypertension was 1.46 (95\% CI 1.11-1.93). Whether the CHNS study assessed the regional difference of the association is unknown. Studies on selenium intake and hypertension in China are limited. A cross-sectional study of 2019 adults in Hunan Province found that selenium intake was inversely associated with metabolic syndrome [24]. However, the association between selenium intake and hypertension was not statistically significant. In a case-control study conducted in Shanghai, including 550 adults, selenium intake was not associated with metabolic syndrome [25]. However, the association between selenium intake and blood pressure was not assessed.

The interaction between region and selenium intake to hypertension is interesting. Such interaction may explain the inconsistent findings on the association between selenium intake and hypertension in studies conducted outside the country. Dietary intake is the primary way for humans to consume selenium. The level of selenium is positively correlated with the protein content of the food. Selenate in plants needs to be converted into an organic form before it can be consumed by humans [26]. Selenium in foods (both animal and plant derived) is highly dependent on the amount of selenium in the environment $[8,26]$. Selenium soil levels are highly correlated with blood selenium levels [27], suggesting the importance of the soil environment in selenium intake. The intake of Selenium around the world varies mostly [4]. For example, the mean selenium content of soils in Enshi (China) is about 150-500 times higher than the average selenium content in selenium-deficient areas in China [28]. Geographically, China has a low selenium belt that stretches from Heilongjiang province in Northeast China to Yunnan Province in the Southeast; this area has a soil selenium content of less than $0.4 \mu \mathrm{g} / \mathrm{g}$ [29]. The difference in the association of selenium intake and hypertension between the north and the south may be related to the fact that the four northern provinces in our study are in the low selenium belt (Supplement Figure S2) [29]. Uncontrolled ingestion of selenium-rich products may result in poisoning [30]. Recommends for selenium vary by geographic region [26]. Our results on regional differences suggested that dietary supplement use should take the region into consideration.

Several mechanisms may explain the inverse association between selenium intake and hypertension in participants from the northern region. First, animal study results have suggested that Selenium inhibits adipocyte hypertrophy and adipogenesis [31]. In a large population study of 3214 adults in Canada, $9-27 \%$ of the change in body fat can be attributed to daily selenium intake $(\mu \mathrm{g} / \mathrm{kg} /$ day), and the body fat percentage decreased by $3-6 \%$ with daily selenium intake increased by $1 \mathrm{~g} / \mathrm{kg} /$ day [32]. In a three-month randomized trial of 27 overweight/obese individuals, selenium supplementation reinforced the effects of diet on obesity and increased lean muscle mass [33]. In China, the prevalence of obesity and hypertension is higher in the north than in the south [34,35]. Second, selenium can reduce inflammation and acquire active immunity by inhibiting the NF-kB signal $[36,37]$. An increasing amount of evidence suggested that immune mechanisms participate in the pathogenesis of hypertension [38].

The positive association between selenium intake and hypertension in participants from the south region is puzzling. While a similar association between serum selenium and hypertension was found in other studies in the USA [39,40], the selenium intake levels are much higher in the USA than in China. Further research is needed to validate our findings.

Our study has several strengths. First, this is a longitudinal study with multiple waves of dietary assessments using three-day food recall, combined with household food inventory records. We used cumulative mean selenium intake in our data analysis to 
reflect the long-term selenium intake [15]. Second, we used trajectory analysis of selenium intake to examine its relationship with hypertension. To our knowledge, this is the first study to report the relationship between the trajectory of selenium intake and hypertension risk. Furthermore, we adjusted for the overall dietary patterns using factor analysis. Third, the relatively long period of follow-up and the large sample size provides sufficient statistical power.

This study has several limitations that need to be acknowledged. First, it was unclear whether long-term intake can be adequately reflected in a three-day diet survey. The survey was not spread over four seasons. Thus, we were unable to adjust for seasonal variations in selenium intake. Second, although we adjusted for income, BMI, and dietary patterns in the multivariable analysis, residual confounding was still possible. Third, the subgroup analyses (e.g., interaction between region and trajectories of selenium intake) were explorative. Furthermore, there was no data on serum selenium.

\section{Conclusions}

In conclusion, selenium intake is relatively stable over the past two decades in the Chinese adults included in the study. Selenium intake was inversely associated with the risk of hypertension in participants from the north, but was positively associated with hypertension in participants from the south. High selenium intake appeared to be a protective factor for blood pressure in the low-selenium region. Thus, dietary supplement use should take the region into consideration.

Supplementary Materials: The following are available online at https: / www.mdpi.com/article / 10.3390/nu13051501/s1, Figure S1: Distribution of selenium intake at baseline among participants in the China Health and Nutrition Survey; Figure S2: The position of provinces investigated on the soil Se distribution map of China. Data source: Sun GX, Meharg AA, Li G, Chen Z, Yang L, Chen SC, Zhu YG. Distribution of soil selenium in China is potentially controlled by deposition and volatilization? Sci Rep. 2016 Feb 17;6:20953. doi:10.1038/srep20953. PMID: 26883576; PMCID: PMC4756323. Compared to the published figure, we removed the legend showing desertification (irrelevant to this study) and added the positioning of nine provinces as well as Beijing and Shanghai. Creative Commons license. http:/ / creativecommons.org/licenses/by/4.0/ (accessed on 9 October 2020); Table S1: Baseline sample characteristics by trajectory of selenium intake: CHNS $(N=10,025)$.

Author Contributions: C.X. and Z.S. contributed to the conception, analysis, and interpretation of data, drafted the report and received the final version for publication. J.X., M.Z., Z.C., S.L., and Y.Z. contributed to analyzing and interpreting the data, commented on the report, revised the manuscript, and approved the final version for publication. All authors have read and agreed to the published version of the manuscript.

Funding: Chongqing Nutrition Society (2019001), RMB 80,000.

Institutional Review Board Statement: Ethical review and approval were waived for this study, due to the use of publicly-available datasets from the official CHNS website (https:/ / www.cpc.unc.edu/ projects / china) (accessed on 9 October 2020).

Informed Consent Statement: Not applicable.

Data Availability Statement: CHNS repository. https://www.cpc.unc.edu/projects/china, accessed date: 15 December 2019.

Acknowledgments: This research uses data from China Health and Nutrition Survey (CHNS). We thank the National Institute for Nutrition and Health, China Center for Disease Control and Prevention, Carolina Population Center (P2C HD050924, T32 HD007168), the University of North Carolina at Chapel Hill, the NIH (R01-HD30880, DK056350, R24 HD050924, and R01-HD38700) and the NIH Fogarty International Center (D43 TW009077, D43 TW007709) for financial support for the CHNS data collection and analysis files from 1989 to 2015 and future surveys, and the China-Japan Friendship Hospital, Ministry of Health for support for CHNS 2009, Chinese National Human Genome Center at Shanghai since 2009, and Beijing Municipal Center for Disease Prevention and Control since 2011. We also express our gratitude to Chongqing Nutrition Society's project 
(2019001) - Research on dietary Selenium intake and its relationship with hypertension and diabetes in middle-aged and older adults in Jiangjin Chongqing-for its support to this study. We are very grateful to Professor Colin for polishing the language of the article.

Conflicts of Interest: We declare that we have no conflict of interest.

\section{Abbreviations}

CHNS: China Health and Nutrition Survey; Se, Selenium.

\section{References}

1. WHO. Global Status Report on Noncommunicable Diseases 2014. Available online: https://apps.who.int/iris/bitstream/handle/ 10665/148114/9789241564854_eng.pdf?sequence=1 (accessed on 9 October 2020).

2. Wang, Z.; Chen, Z.; Zhang, L.; Wang, X.; Hao, G.; Zhang, Z.; Shao, L.; Tian, Y.; Dong, Y.; Zheng, C.; et al. Status of hypertension in China: Results from the china hypertension survey, 2012-2015. Circulation 2018, 137, 2344-2356. [CrossRef] [PubMed]

3. Ndanuko, R.N.; Tapsell, L.C.; Charlton, K.E.; Neale, E.P.; Batterham, M.J. Dietary patterns and blood pressure in adults: A systematic review and meta-analysis of randomized controlled trials. Adv. Nutr. 2016, 7, 76-89. [CrossRef] [PubMed]

4. Rayman, M.P. Selenium and human health. Lancet 2012, 379, 1256-1268. [CrossRef]

5. Kieliszek, M.; Błażejak, S. Current knowledge on the importance of selenium in food for living organisms: A review. Molecules 2016, 21, 609. [CrossRef]

6. Schomburg, L. Dietary selenium and human health. Nutrients 2016, 9, 22. [CrossRef]

7. Jones, G.D.; Droz, B.; Greve, P.; Gottschalk, P.; Poffet, D.; McGrath, S.P.; Seneviratne, S.I.; Smith, P.; Winkel, L.H. Selenium deficiency risk predicted to increase under future climate change. Proc. Natl. Acad. Sci. USA 2017, 114, 2848-2853. [CrossRef]

8. Li, S.; Bañuelos, G.S.; Wu, L.; Shi, W. The changing selenium nutritional status of Chinese residents. Nutrients 2014, 6, 1103-1114. [CrossRef]

9. Benstoem, C.; Goetzenich, A.; Kraemer, S.; Borosch, S.; Manzanares, W.; Hardy, G.; Stoppe, C. Selenium and its supplementation in cardiovascular disease-What do we know? Nutrients 2015, 7, 3094-3118. [CrossRef]

10. Loscalzo, J. Keshan disease, selenium deficiency, and the selenoproteome. N. Engl. J. Med. 2014, 370, 1756-1760. [CrossRef]

11. Kuruppu, D.; Hendrie, H.C.; Yang, L.; Gao, S. Selenium levels and hypertension: A systematic review of the literature. Public Health Nutr. 2014, 17, 1342-1352. [CrossRef]

12. Retondario, A.; Fernandes, R.; Rockenbach, G.; Alves, M.A.; Bricarello, L.P.; Trindade, E.; Vasconcelos, F.A.G. Selenium intake and metabolic syndrome: A systematic review. Clin. Nutr. 2019, 38, 603-614. [CrossRef]

13. MacFarquhar, J.K.; Broussard, D.L.; Melstrom, P.; Hutchinson, R.; Wolkin, A.; Martin, C.; Burk, R.F.; Dunn, J.R.; Green, A.L.; Hammond, R.; et al. Acute selenium toxicity associated with a dietary supplement. Arch. Intern. Med. 2010, 170, $256-261$. [CrossRef]

14. Yang, Y. China Food Composition Tables (Standard Edition), 6th ed.; Peking University Medical Press: Beijing, China, 2018 ; Volume 1.

15. Hu, F.B.; Stampfer, M.J.; Rimm, E.; Ascherio, A.; Rosner, B.A.; Spiegelman, D.; Willett, W.C. Dietary fat and coronary heart disease: A comparison of approaches for adjusting for total energy intake and modeling repeated dietary measurements. Am. J. Epidemiol. 1999, 149, 531-540. [CrossRef]

16. Zhai, F.Y.; Du, S.F.; Wang, Z.H.; Zhang, J.G.; Du, W.W.; Popkin, B.M. Dynamics of the Chinese diet and the role of urbanicity, 1991-2011. Obes. Rev. 2014, 15, 16-26. [CrossRef]

17. Zhou, B.F. Effect of body mass index on all-cause mortality and incidence of cardiovascular diseases-Report for meta-analysis of prospective studies open optimal cut-off points of body mass index in Chinese adults. Biomed. Environ. Sci. BES 2002, 15, 245-252.

18. Zhou, B.F. Predictive values of body mass index and waist circumference for risk factors of certain related diseases in Chinese adults-Study on optimal cut-off points of body mass index and waist circumference in Chinese adults. Biomed. Environ. Sci. BES 2002, 15, 83-96.

19. Shi, Z.; Taylor, A.W.; Riley, M.; Byles, J.; Liu, J.; Noakes, M. Association between dietary patterns, cadmium intake and chronic kidney disease among adults. Clin. Nutr. 2018, 37, 276-284. [CrossRef]

20. Chinese Nutrition Society. Chinese DRIs Handbook; Standards Press of China: Beijing, China, 2013.

21. Nawrot, T.S.; Staessen, J.A.; Roels, H.A.; Den Hond, E.; Thijs, L.; Fagard, R.H.; Dominiczak, A.F.; Struijker-Boudier, H.A. Blood pressure and blood selenium: A cross-sectional and longitudinal population study. Eur. Heart J. 2007, 28, 628-633. [CrossRef]

22. Su, L.; Jin, Y.; Unverzagt, F.W.; Liang, C.; Cheng, Y.; Hake, A.M.; Kuruppu, D.; Ma, F.; Liu, J.; Chen, C.; et al. Longitudinal association between selenium levels and hypertension in a rural elderly Chinese cohort. J. Nutr. Health Aging 2016, 20,983-988. [CrossRef]

23. Liu, L.; Lin, G.; Wang, H.; Zhang, B.; Du, S. Selenium exposure and incident hypertension among Chinese adults (P24-020-19). Curr. Dev. Nutr. 2019, 3. [CrossRef]

24. Wei, J.; Zeng, C.; Gong, Q.Y.; Li, X.X.; Lei, G.H.; Yang, T.B. Associations between dietary antioxidant intake and metabolic syndrome. PLoS ONE 2015, 10, e0130876. [CrossRef] [PubMed] 
25. Li, Y.; Guo, H.; Wu, M.; Liu, M. Serum and dietary antioxidant status is associated with lower prevalence of the metabolic syndrome in a study in Shanghai, China. Asia Pac. J. Clin. Nutr. 2013, 22, 60-68. [CrossRef] [PubMed]

26. Kieliszek, M. Selenium-fascinating microelement, properties and sources in food. Molecules 2019, 24, 1298. [CrossRef] [PubMed]

27. Thomson, C.D. Assessment of requirements for selenium and adequacy of selenium status: A review. Eur. J. Clin. Nutr. 2004, 58, 391-402. [CrossRef]

28. Yuan, L.; Yin, X.; Zhu, Y.; Li, F.; Huang, Y.; Liu, Y.; Lin, Z. Selenium in plants and soils, and selenosis in Enshi, China: Implications for selenium biofortification. In Phytoremediation and Biofortification: Two Sides of One Coin; Yin, X., Yuan, L., Eds.; Springer: Dordrecht, The Netherlands, 2012; pp. 7-31. [CrossRef]

29. Sun, G.-X.; Meharg, A.A.; Li, G.; Chen, Z.; Yang, L.; Chen, S.-C.; Zhu, Y.-G. Distribution of soil selenium in China is potentially controlled by deposition and volatilization? Sci. Rep. 2016, 6, 20953. [CrossRef]

30. Zagrodzki, P.; Laszczyk, P. Selenium and cardiovascular disease: Selected issues. Postepy Hig. I Med. Dosw. 2006, 60, 624-631.

31. Kim, J.E.; Choi, S.I.; Lee, H.R.; Hwang, I.S.; Lee, Y.J.; An, B.S.; Lee, S.H.; Kim, H.J.; Kang, B.C.; Hwang, D.Y. Selenium significantly inhibits adipocyte hypertrophy and abdominal fat accumulation in OLETF rats via induction of fatty acid beta-oxidation. Biol. Trace Elem. Res. 2012, 150, 360-370. [CrossRef]

32. Wang, Y.; Gao, X.; Pedram, P.; Shahidi, M.; Du, J.; Yi, Y.; Gulliver, W.; Zhang, H.; Sun, G. Significant beneficial association of high dietary selenium intake with reduced body fat in the coding study. Nutrients 2016, 8, 24. [CrossRef]

33. Cavedon, E.; Manso, J.; Negro, I.; Censi, S.; Serra, R.; Busetto, L.; Vettor, R.; Plebani, M.; Pezzani, R.; Nacamulli, D.; et al. Selenium supplementation, body mass composition, and leptin levels in patients with obesity on a balanced mildly hypocaloric diet: A pilot study. Int. J. Endocrinol. 2020, 2020, 4802739. [CrossRef]

34. Pei, L.; Wu, J.; Wang, Z.; Wang, X.; Chen, Z.; Li, J.; Zhang, L.; Fan, R.; Zhang, Z.; Dong, Y.; et al. Geographic variations and potential macro-environmental exposure of hypertension: From the China hypertension survey. J. Hypertens. 2020, 38, 829-838. [CrossRef]

35. Zhang, J.G.; Wang, Z.H.; Wang, H.J.; Du, W.W.; Su, C.; Zhang, J.; Jiang, H.R.; Zhai, F.Y.; Zhang, B. Dietary patterns and their associations with general obesity and abdominal obesity among young Chinese women. Eur. J. Clin. Nutr. 2015, 69, 1009-1014. [CrossRef]

36. Kaushal, N.; Kudva, A.K.; Patterson, A.D.; Chiaro, C.; Kennett, M.J.; Desai, D.; Amin, S.; Carlson, B.A.; Cantorna, M.T.; Prabhu, K.S. Crucial role of macrophage selenoproteins in experimental colitis. J. Immunol. 2014, 193, 3683-3692. [CrossRef]

37. Fairweather-Tait, S.J.; Bao, Y.; Broadley, M.R.; Collings, R.; Ford, D.; Hesketh, J.E.; Hurst, R. Selenium in human health and disease. Antioxid. Redox Signal. 2011, 14, 1337-1383. [CrossRef]

38. Rodriguez-Iturbe, B.; Pons, H.; Johnson, R.J. Role of the immune system in hypertension. Physiol. Rev. 2017, 97, 1127-1164. [CrossRef]

39. Bastola, M.M.; Locatis, C.; Maisiak, R.; Fontelo, P. Selenium, copper, zinc and hypertension: An analysis of the National Health and Nutrition Examination Survey (2011-2016). BMC Cardiovasc. Disord. 2020, 20, 45. [CrossRef]

40. Laclaustra, M.; Navas-Acien, A.; Stranges, S.; Ordovas, J.M.; Guallar, E. Serum selenium concentrations and hypertension in the US Population. Circ. Cardiovasc. Qual. Outcomes 2009, 2, 369-376. [CrossRef] 